\title{
Optical countermeasures against CLOS weapon systems
}

\author{
Alexander Toet ${ }^{\mathrm{a}}$, Koen W. Benoist ${ }^{\mathrm{b}}$, Joost N.J. van Lingen ${ }^{\mathrm{c}}$, H. (Ric) M.A. Schleijpen ${ }^{\mathrm{b}}$ \\ ${ }^{\mathrm{a}} \mathrm{TNO}$, Kampweg 5, 3769DE Soesterberg, The Netherlands \\ ${ }^{\mathrm{b}}$ TNO, Oude Waalsdorperweg 63, 2597 AK The Hague, The Netherlands \\ ${ }^{\mathrm{c}}$ TNO, Ypenburgse Boslaan 2, 2496 ZA The Hague, The Netherlands
}

\begin{abstract}
There are many weapon systems in which a human operator acquires a target, tracks it and designates it. Optical countermeasures against this type of systems deny the operator the possibility to fulfill this visual task. We describe the different effects that result from stimulation of the human visual system with high intensity (visible) light, and the associated potential operational impact. Of practical use are flash blindness, where an intense flash of light produces a temporary "blind-spot" in (part of) the visual field, flicker distraction, where strong intensity and/or color changes at a discomfortable frequency are produced, and disability glare where a source of light leads to contrast reduction. Hence there are three possibilities to disrupt the visual task of an operator with optical countermeasures such as flares or lasers or a combination of these; namely, by an intense flash of light, by an annoying light flicker or by a glare source. A variety of flares for this purpose is now available or under development: high intensity flash flares, continuous burning flares or strobe flares which have an oscillating intensity. The use of flare arrays seems particularly promising as an optical countermeasure. Lasers are particularly suited to interfere with human vision, because they can easily be varied in intensity, color and size, but they have to be directed at the (human) target, and issues like pointing and eye-safety have to be taken into account. Here we discuss the design issues and the operational impact of optical countermeasures against human operators.
\end{abstract}

Keywords: non-lethal weapons, flare countermeasures, lasers.

\section{INTRODUCTION}

Many weapon systems involve a human operator to acquire and track a target. A specific example are the CLOS (Command to line of Sight) guided weapons. In this type of guidance the operator tracks the target during the missile flight, either through an optical sight in the visible range or through a thermal sight. In contrast to "fire and forget" weapons the countermeasures (CM) for the type of weapon systems described above should be effective against a human operator instead of an automatic tracker. The optical countermeasures should deny the operator to see the target or interfere in another way with the tracking ability of the operator.

In this paper we investigate the possible effects of optical countermeasures - flares or lasers or a combination of these on a human operator. We will pay special attention to the robustness of these effects, since the practical value of a countermeasure critically depends on its reliability to achieve the desired effect. A wide variety of lasers is currently available that may be deployed for optical countermeasures, and several dedicated systems have been developed in recent years. Several types of flares which are suitable for this purpose have also become available or are currently under development, like high intensity flash flares, continuous burning flares or strobe flares which have an oscillating intensity. We first present the results of a literature study that was performed to investigate the possible effects of various optical countermeasures on human observers. Next we will assess the robustness of these effects. Optical effects which affect only a limited part of the population cannot be used as a reliable countermeasure. Optical effects which can be mitigated by operator training will also be less suitable as countermeasures. Then we will discuss how the most reliable of these effects can be translated into countermeasure requirements for interference with human weapon operators. The main questions in this study are: "What optical countermeasures are needed to reliably deny the operator the ability to track a target, and what are the available light sources that can produce these measures?"

Technologies for Optical Countermeasures X; and High-Power Lasers 2013: Technology and Systems, edited by David H. Titterton, Mark A. Richardson, Robert J. Grasso, Harro Ackermann, Willy L. Bohn,

Proc. of SPIE Vol. 8898, 88980L · C 2013 SPIE · CCC code: 0277-786X/13/\$18 - doi: 10.1117/12.2028342 


\section{EFFECTS OF VISIBLE LIGHT ON HUMAN OBSERVERS}

Light induced visual disturbances are the result of neural processing in the retina or in areas upstream the visual cortex, and thus occur only after light has been absorbed by the photoreceptors. For the process of light absorption it does not matter whether the light originates from a laser, a flare or an ordinary light source like the sun or a searchlight. Therefore, in the following discussion of effects, the light source does not really matter.

The (physiological and psychological) effects induced by ocular exposures to wavelengths of light that are absorbed by the retina of the eye - which are primarily in the near-infrared and the visible portions of the electromagnetic spectrum - can be distinguished into a number of general categories ${ }^{1}$. Table 1 provides an overview, in which primary and secondary effects are listed.

Table 1. Physiological and psychological effects elicited by light-stimulation.

\begin{tabular}{|c|c|c|c|}
\hline \multicolumn{2}{|l|}{ Type } & Primary effect & Secondary effect \\
\hline \multirow{5}{*}{ Single flash } & \multirow{3}{*}{$\begin{array}{l}\text { No biological } \\
\text { damage, complete } \\
\text { recovery with time, } \\
\text { reversible }\end{array}$} & Distraction & \multirow{6}{*}{$\begin{array}{l}\text { startle, fear, } \\
\text { demotivation }\end{array}$} \\
\hline & & Glare & \\
\hline & & Flash blindness & \\
\hline & \multirow{2}{*}{$\begin{array}{l}\text { Biological damage, } \\
\text { irreversible }\end{array}$} & Thermal lesions & \\
\hline & & Haemorrhagic lesions & \\
\hline Multiple flashes & & Photosensitivity & \\
\hline
\end{tabular}

\subsection{Reversible effects}

The unexpected occurrence of a flash of light in a scene inherently attracts visual attention and will distract someone from his actual task ${ }^{2}$. A secondary effect may be startle or fear reactions, the magnitude of which may be expected to be partly reduced by habituation and desensitization (training).

Glare (sometimes called dazzle) refers to the temporary inability to see details in the area of the visual field around a bright light (such as an oncoming car's headlights). Glare is not associated with biological damage. It lasts only as long as the bright light is actually present within the individual's field of vision. Laser glare can be more intense than solar glare. But also, in dark surroundings, low levels of laser light may already cause inconvenient glare. Glare that impairs vision is called disability glare.

Disability glare is primarily caused by the diffractions and scattering of light inside the eye due to the imperfect transparency of the optical components of the eye and to a lesser extent by diffuse light passing through the scleral wall or the iris ${ }^{3}$. The scattered light overlays the retinal image, thus reducing visual contrast. This overlaying scattered light distribution is usually described as a veiling luminance. This veiling luminance in the human eye reduces the contrast in the scene. The veiling luminance in the eye caused by a point source can be described by the classical Stiles-Holladay equation ${ }^{4,5}$ :

$$
L_{v}=\frac{10 E_{g l}}{\theta^{2}} \quad 3<\theta<30 \mathrm{deg}
$$

where $L_{v}$ is the glare (veiling) luminance $\left(\mathrm{cd} / \mathrm{m}^{2}\right), \mathrm{E}_{\mathrm{gl}}$ represents the illuminance at the eye (in lx) by the laser, and $\theta$ the glare angle (in degrees). For smaller angles the equation had been refined as follows ${ }^{6}$ :

$$
L_{v}=10 E_{g l}\left(\frac{1}{(\theta+0.2)^{2}}+\frac{1}{(\theta+0.2)^{3}}\right) \quad 0.17<\theta<30 \mathrm{deg}
$$


Flash blindness is similar in effect to having the eyes exposed to a camera flashlight. It is a temporary loss of vision produced when retinal light-sensitive pigments are bleached by light more intense than that to which the retina is physiologically adapted at that moment. An afterimage, which moves with the eye, persists for several seconds to several minutes after the light source is turned off. This afterimage produces a temporary scotoma (blind spot) in the visual field in which targets are either partially or completely obscured. The time required for temporary flash blindness-induced scotomas to fade increases with the brightness and duration of the light insult. The time it takes before the ability to perceive targets returns depends on several factors, including target contrast, brightness, color, size, observer age, and the overall adaptation state of the visual system ${ }^{7-12}$. Complete dark adaptation of the visual system takes 20 to 30 minutes $^{13}$ whereas the opposite (adaptation to an environment of bright light) is usually completed within 2 minutes ${ }^{14}$. So, under scotopic conditions (night time light levels), flash blindness will be most drastic and easiest to achieve.

\subsection{Irreversible effects (permanent damage)}

Glare and flash blindness are temporary bio-effects, but they can accompany permanent bio-effects, that is thermal and haemorrhagic lesions ${ }^{15,16}$. Thermal lesions are burns of the retinal tissue that result in permanent scotomas. Haemorrhagic lesions are ruptures of the retinal and sub-retinal blood vessels resulting from thermo-acoustical shockwaves induced in the eye by laser pulses. Simply stated, the light source deposits energy into the eye, which rapidly heats up and produces a shock wave due to the expansion of the vitreous humour which tears the thin photoreceptor layer of the retina. Lesions can produce immediate and severe permanent visual disruption ${ }^{17}$.

In the rest of this study we will not consider optical countermeasures that may cause irreversible effects since their use is restricted according to Protocol IV of the Geneva Convention ${ }^{18}$.

\subsection{Luminance flicker}

Studies on the visual effects due to dynamic changes in light level reveal a number of interesting facts ${ }^{19-22}$. Flickering lights within the frequency range $2-25 \mathrm{~Hz}$ are perceived as disturbing. At $10 \mathrm{~Hz}$ the subjective brightness of flickering lights is at maximum, known as the Brucke-Bartley effect ${ }^{23}$. The rate of discomfort depends on the modulation depth and the intensity time profile of the flicker. The modulation depth is defined as the difference between the maximum and minimum light level ${ }^{24}$. The shape of the intensity profile with time also determines effectiveness of the flicker: short flashes in which the duration of the ON-cycle is less than $25 \%$ of the total ON-OFF cycle (the so called pulse-to-cycle ratio $^{24}$ ) are visually most effective. Perceived discomfort also depends on the size of the light source: the larger the visual angle of the light source in the visual field, the more discomfort is experienced. However, this is only true when the intensity of the light source is kept constant. When keeping retinal illuminance (i.e., the amount of light falling upon the eye) fixed the discomfort increases with decreasing light source $\operatorname{area}^{25,26}$.

Luminance flicker (temporal intensity modulations of bright lights) can trigger additional adverse physiological and psychological symptoms, ranging from vertigo, disorientation, mild headaches and muscle spasm to convulsions or epileptic seizures. These effects increase with the intensity of the source and are usually stronger when the light is spatially scanning through a pattern ${ }^{29}$. Intensities of 0.2-1.5 million candlepower (cd) are in the range capable to trigger seizures. Bright and flickering light sources that cover the majority of the visual field are most effective in disrupting the normal brain activity. During a seizure, nerve cells in the brain fire electrical impulses at a rate of up to four times higher than normal, causing a sort of electrical storm in the brain. This may lead to epileptic seizures in persons sensitive to this type of stimulation (photosensitivity or photosensitive epilepsy ${ }^{30}$ ). Sleep deprivation and prolonged exposure may increase seizure occurrence ${ }^{31,32}$. However, the estimated prevalence of seizures from light stimuli is approximately 1 per 10,000 , or 1 per 4,000 individuals with an age between 5-24 years ${ }^{33}$, therefore this effect will not be considered in this study as a potential countermeasure against CLOS operators.

Recent studies have shown that dynamic stimulation with intense bright light at frequencies which are considered optimally disruptive has no effect on human tracking performance ${ }^{27,28}$, even when the visual experience is subjectively rated as being "unbearable". 


\subsection{Chromatic flicker}

Chromatic flicker (temporal chromaticity modulations of bright lights) can trigger sustained cortical excitation even in normal subjects, which is largest at a driving frequency of $10 \mathrm{~Hz}$, and strongest for Red/Blue flicker, followed by Blue/Green and Red/Green ${ }^{34,35}$. Red-blue flicker is most provocative below $30 \mathrm{~Hz}^{36-38}$. The induced cortical excitation manifests itself in a chromatic flash VEP (visually evoked potential), which is the primary response of the target to the chromatic flicker and can be measured on a EEG (electroencephalogram). The chromatic flash VEP has separate color and luminance components ${ }^{39}$. Flicker VEPs elicited by different color and luminance combinations at modulating stimulus frequencies between 3 and $24 \mathrm{~Hz}$ show peaks at $9 \mathrm{~Hz}$ for luminance flicker and at $18 \mathrm{~Hz}$ for color flicker ${ }^{40}$.

Recently powerful compact multicolored lights have been designed as non-lethal weapons ${ }^{41,42}$. These devices deploy chromatic strobing patterns with frequencies that are claimed to induce sustained cortical excitation even in normal subjects $^{43}$. The inventors claim that these devices induce disorientation, dizziness, and vertigo, and that "there is one combination of wavelengths and frequency that seems to affect just about everyone"43. However, all reports on the effectiveness of these devices are still anecdotal. Neurologists and other medical experts are indeed skeptical of the notion that flashing lights can have any robust and profound influence on the brain. Until this time there are only few controlled and systematic studies in this area. TNO recently investigated human performance in driving, search and tracking tasks in conditions with intense flicker, for a range of different (a-)chromatic and temporal flicker patterns ${ }^{27}$. It was found that driving and tracking performance were not affected by (a-)chormatic flicker even up to associated levels of glare that were subjectively rated 'unbearable'. Search times increased in conditions with flicker, but chromatic flicker did not disturb performance more than achromatic flicker (there was even a tendency that achromatic flicker appeared more effective, although this effect was not significant). These results agree with earlier findings that bright light insults have no effect on tracking performance ${ }^{28}$, and that strobing lights are not more effective in disrupting tracking performance than continuous stimulation ${ }^{44}$.

Since it has not been possible to reliably demonstrate the capability of chromatic flicker to disturb human visual task performance for this effect will not be considered any further in this study.

\section{FACTORS AFFECTING THE EFFECTIVENESS OF OPTICAL COUNTERMEASURES}

This section describes the counter-countermeasures which can be taken by an operator to mitigate the optical countermeasure effects.

\subsection{Physiological protection mechanisms}

The human eye is to a limited extent capable of protecting itself against an overdose of light. We distinguish four mechanisms. First of all, a pupillary response will cause the pupil to constrict about after the onset of a flash of light, thereby attenuating the total energy entering the eye. Pupil constriction has a latency of about $100 \mathrm{~ms}$ and the pupil diameter reaches a minimum after approximately $1 \mathrm{~s}$ for brief $(0.1$ to $1 \mathrm{~s})$ exposures while it continues to decrease up to $3 \mathrm{~s}$ at longer exposure durations ${ }^{44}$. However, since pupil size may only vary from about 8 to $2 \mathrm{~mm}$ (diameter), the reduction in retinal illuminance by contraction of the pupil is only a factor of $\left(4^{2}=\right) 16$. Second, an eyeblink response (startle reflex) will occur after about $100 \mathrm{~ms}^{28}$, although $250 \mathrm{~ms}$ has also been reported ${ }^{45}$. These two mechanisms (blink and pupillary restriction) serve to attenuate the total energy entering the eye. The third protection mechanism of the eye is to adapt its sensitivity by reorganizing the nerve cells in the retina (transient adaptation). Though this process is relatively fast (occurring within about $200 \mathrm{~ms}$ ), it is not suited to counter large changes in luminance. Fourth, in the case of more dramatic changes in luminance, photochemical adaptation can take place. Here, pigments in the rods and cones are bleached or regenerated resulting in adaptation to changes in brightness. While bleaching occurs almost instantaneous, adaptation mechanisms are much slower (2 minutes for adapting to a bright environment, 20-30 minutes for adapting to the dark).

\subsection{Technological protection measures}

There are also several technological measures available to protect the human eye from damage by high intensity light (for an overview $\mathrm{se}^{46}$ ). Simple wide band filters (e.g., sunglasses) can effectively reduce the perceived intensity of the light source, but also the overall contrast in a scene, especially at night. 
Fixed line spectral filters can effectively be deployed against lasers with known fixed frequencies. These filters inevitably introduce significant visual $\operatorname{loss}^{47}$. Thin-film filters must exhibit sufficient rejection bandwidth to ensure protection at all angles of incidence, and few absorptive filters have rejection bands narrower than $100 \mathrm{~nm}$. Even if the protection only addresses frequency-doubled Nd:YAG lasers, the total transmission is generally less than $60 \%$.

Frequency agile filters can be deployed to counter unknown laser types or lasers with time varying frequencies. However, protective systems incorporating suitable glasses or visors holding frequency agile filters of this kind however, are not commercially available today.

Active systems like shutters, frequency agile filters or spatial light modulators used to block light sources have a finite response time and are therefore useless against short laser pulses.

\subsection{Ambient conditions}

In bright daylight the laser light intensity has to compete with the light intensity produced by the sun. Dark ambient conditions increase the capability to dazzle observers.

Apart from visibility conditions to be permissible for aiming the laser (e.g., obscuration), unfavorable weather conditions, such as rain, snow, fog and sand storms, dust and smoke may strongly degrade the laser beam intensity effectively delivered to the target person and may even totally block the effect. However, this may not be an issue since the CLOS operator will have difficulties tracking his target anyway in these conditions. Studies on laser dazzling systems have in fact shown that lasers are only effective at night against observers who are looking straight at the laser (i.e., when the light source is in the central visual field of the observer and close to the target fixated by the observer as opposed to many degrees away) and at short exposure distances ${ }^{2}$.

\section{REQUIREMENTS FOR EFFECTIVE OPTICAL COUNTERMEASURES}

From the literature reviewed in Section 2 we identify three types of potential robust optical countermeasures:

1. a high intensity flash - causing temporary flash blindness,

2. a bright light source - causing contrast reduction,

3. a flickering pattern of light - causing contrast reduction.

In the next sections we will discuss the requirements for effective and robust deployment of each of these approaches.

\subsection{Intensity level}

The intensity levels that are needed to effectively deploy the different countermeasures depend on the desired effects. For flash blindness, the intensity of a flash required to obtain a certain recovery time depends on luminance of the light source, background luminance (pupil size and initial adaptation state of the observer), and target-background contrast. For flicker, the degree of discomfort depends on the modulation depth (difference between maximum and minimum light intensity). Maximum applied intensity should of course be below the level at which permanent damage can occur.

In contrast to the effects of lasers, the effects of other high intensity lights on human vision in low ambient light conditions have not been sufficiently investigated. As a result there are currently no well-defined threshold illuminance levels corresponding to the different visual interference effects that may occur when a person is confronted with a high intensity light while performing a complex task (e.g., driving a vehicle). The Society of Automotive Engineers (SAE) therefore proposed to adopt the existing laser safety zoning for critical tasks (e.g., flying an airplane) also for other high intensity light sources and to convert the corresponding maximum admissible irradiance exposure levels to values that apply to broadband high intensity lights ${ }^{48}$.

The ANSI Z136.6 standard defines laser irradiance $\left(\mathrm{W} / \mathrm{cm}^{2}\right)$ threshold exposure levels for visual interference at dusk or at night ${ }^{49}$. When applied to broadband high intensity lights, the appropriate corresponding measure is illuminance $\left(\mathrm{lm} / \mathrm{cm}^{2}\right)$. The laser irradiance threshold levels corresponding to the different visual interference effects can be converted into illuminance threshold levels through multiplication by the luminous efficacy at the peak photopic response, which is $683 \mathrm{~lm} / \mathrm{W}$ at $555 \mathrm{~nm}^{48,50}$. Note that this conversion is based on the assumption that photopic vision applies. The SAE 
argues that this assumption is reasonable when applied to pilots, because the aviator's eyes are generally not entirely dark adapted at night due to ambient lighting, such as illuminated cockpit gauges and ground lights ${ }^{48}$. The same argument probably holds for driving a vehicle at night. Further studies may be required to assess the validity of this assumption. The resulting relationship between broadband illuminance and laser irradiance is shown in Table2.

Table 2. Broadband illuminance threshold exposure levels, and corresponding laser irradiance thresholds for different visual effects, as defined by ANSI Z136.6 (American National Standards Institute, 2005, Table 5).

\begin{tabular}{|lcc|}
\hline Visual effect & Irradiance threshold $\left[\mathrm{W} / \mathrm{cm}^{2}\right]$ & Illuminance threshold [Ix] \\
\hline Eye damage & $2.5 \times 10^{-3}$ & 17,000 \\
Afterimages, flashblindness & $1 \times 10^{-4}$ & 680 \\
Glare & $5 \times 10^{-6}$ & 34 \\
Startle, distraction & $5 \times 10^{-8}$ & 0.34 \\
\hline
\end{tabular}

We will use the values in Table 2 as guidance for the requirements for the intensity levels required to achieve effective optical countermeasures.

\subsection{Optimum wavelength}

The following facts from the literature suggest that there may be a wavelength that is optimal for inducing visual disturbance:

- Optimal sensitivity of the eye during daytime (photopic vision using cones) is at $555 \mathrm{~nm}$ (yellow-green), and $505 \mathrm{~nm}$ (blue-green) at night (scotopic vision with rods) ${ }^{51}$.

- At shorter wavelengths - towards the blue end of the spectrum $(350-450 \mathrm{~nm})$ - absorbance by the lens causes fluorescence which in turn produces intraocular veiling glare $(480-520 \mathrm{~nm})^{51}$.

- Readaptation time to flashes with different wavelength is longest for $520 \mathrm{~nm}$ green ${ }^{10}$. The readaptation time was measured using optokinetic nystagmus elicited by a projected moving striped pattern in a hemisphere ${ }^{51}$.

- Studies on the disturbing effects of laser induced glare of different wavelengths ( $364 \mathrm{~nm}$, ultra-violet; $405 \mathrm{~nm}$, violet; $532 \mathrm{~nm}$, green and $647 \mathrm{~nm}$, red) on visual search show an increased search time with $532 \mathrm{~nm}^{51}$.

- Monochromatic green laser light causes more disability glare than either alternating red-green or polychromatic exposure ${ }^{52}$.

- Green light more effectively disrupts tracking performance than red light ${ }^{44}$.

- Operators use the central part of their visual field (the fovea) in which cone vision dominates to accurately track targets. For the detection and tracking of small objects, the L and M-cones with peak sensitivity at 530 and 560 $\mathrm{nm}$, respectively, are most important. This implies that for maximum interference with the operator's task, both the L and M cones should be "attacked". A single wavelength of $545 \mathrm{~nm}$ (halfway in between 530 and $560 \mathrm{~nm}$ ) would be optimally suited to achieve this goal.

Hence, the literature suggests that a wavelength of $545 \mathrm{~nm}$ is optimal for inducing flash blindness. It will simultaneously affect the $\mathrm{L}$ and $\mathrm{M}$ cones that are required for target tracking.

\subsection{Flash duration}

Pupillary occlusion or constriction cannot reduce the retinal exposure for exposure durations below $70 \mathrm{~ms}^{44}$. Most active protection devices also typically have latencies longer than $100 \mathrm{~ms}$. Hence, the amount of energy required to elicit the desired visual effect should be delivered to the eye within $100 \mathrm{~ms}$.

\subsection{Pulse frequency}

Frequency should be between 5 and $15 \mathrm{~Hz}$ for maximum discomfort.

\subsection{Pulse-to-cycle ratio}

The transitions from dark to bright (and vice versa) should be as fast and strong as possible to induce maximum discomfort. In addition, the pulse-to-cycle ratio should be 0.25 or less. 


\subsection{Angular extent}

The impact of a light source will be maximal when it covers the entire visual field of the operator. However, to effectively reduce the visual abilities of an operator it is probably sufficient if the light induces a temporary "blindness" in the central part of the visual field (the fovea), with an extent of about 2-4 degrees.

\subsection{Effect on viewing through optical sights}

The use of optical sights by CLOS weapon operators will increase the irradiance of the eye by the countermeasure compared to the irradiance of the unaided eye. In principle the irradiance from a point source will increase by $\mathrm{M}^{2}$, where $\mathrm{M}$ is the magnifying power of the optical system. Transmission losses in the optics will reduce this enhancement. From the countermeasure requirement point of view the use of optical sights will relax rather than increase the countermeasure source intensity requirement.

\subsection{Time-of-day}

The larger pupil size at night (about $7 \mathrm{~mm}$ diameter) allows more light to enter the eye than during daytime (about 3 $\mathrm{mm}$ ). For instance, in daytime, the dazzling glare extends to $300 \mathrm{~m}$ for a $125 \mathrm{~mW}$ green class 3B laser and to $500 \mathrm{~m}$ for a $200 \mathrm{~mW}$ model ${ }^{53}$. At night, those range double.

Re-adaptation to the dark may take some $20 \mathrm{~min}$, so the effects of afterimages will have more impact than during daytime. Griffioen et al. ${ }^{54}$ calculated that the intensity of a light flash needed to produce a recovery time of 5 seconds during daytime is 1,000 times higher than at night.

From a countermeasure requirement point of view the change from day to night conditions will relax rather than increase the countermeasure source intensity requirement, so the day time requirement will be leading.

\section{DEPLOYMENT ISSUES}

\subsection{Requirements for effective laser countermeasures}

Lasers are highly flexible bright light sources which can easily be built to fulfill all the requirements listed in Section 4 on source intensity, wavelength, modulation frequency and pulse duty cycle. The two main issues for application of lasers are on the one hand the fact that the narrow laser beam needs to be pointed towards the CLOS weapon operator and on the other hand the requirement to avoid permanent damage to the eyes of the operator.

Pointing of the laser beam requires detection and tracing of the operator position. Solutions could range from as simple as a person pointing a handheld laser-dazzler to a DIRCM (directed infrared countermeasure systems) like automatic system. DIRCM systems are primarily built to counter IR guided missiles and include a detection and tracking component and a laser pointing device. Recently DIRCM system concepts have been presented with provision for an additional optical laser channel. Detailed discussion of laser beam pointing is beyond the scope of this paper.

To conform to Protocol IV of the Geneva Convention ${ }^{18}$ optical countermeasures involving lasers should take appropriate measures to prevent permanent damage to the naked eye. Therefore, the laser power should be restricted and the system design should include sufficient safety measures. In the following we will use the Maximum Permissible Exposure (MPE) as defined in international laser safety standards ${ }^{55,56}$. The Nominal Ocular Hazard Distance (NOHD) is the distance from a laser beyond which the MPE is not exceeded. Within the NOHD, the MPE is exceeded and damage can be expected. The MPE is about ten times lower than the laser power level where the first eye-damage (scotomas) occurs. Since visible radiation is required to disrupt direct-sight human vision, the laser safety MPE for continuous visible wavelength $(400-700 \mathrm{~nm})$ lasers should be used:

$$
\text { MPE }=18 t^{0.75} \quad\left[\mathrm{~J} / \mathrm{m}^{2}\right] \quad ; 1.8 \times 10^{-5}<t<10 \mathrm{~s}
$$

where $t$ is the duration of the exposure (in seconds). For direct ocular exposure and a blink reflex with a duration of 250 $\mathrm{ms}$ the MPE is $6.36 \mathrm{~J} / \mathrm{m}^{2}$ which is equivalent to a maximum permissible power density of $\left[6.36 \mathrm{~J} / \mathrm{m}^{2}\right] /\left[250 \times 10^{-3} \mathrm{~s}\right]=25$ $\mathrm{W} / \mathrm{m}^{2}$.

However, since trained operators may be able to suppress the blink reflex, eye-damage may occur even at the MPE. Pulsed lasers may also be used to counter the blink reflex, and may also cause additional startle and distraction. 


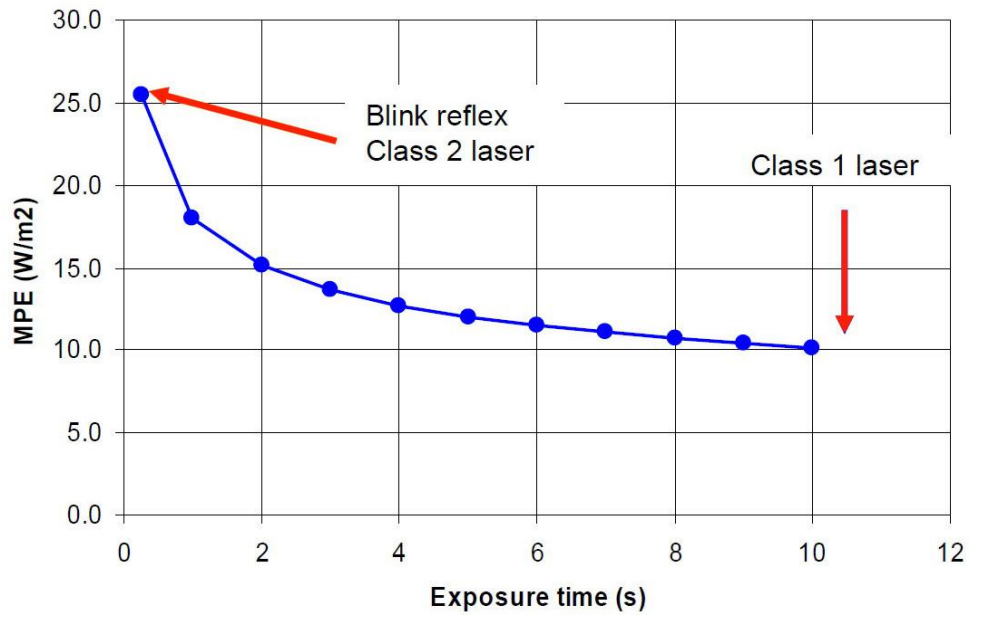

Figure 1. Maximum permissible exposure as a function of exposure time for continuous lasers at wavelengths 400-700 $\mathrm{nm}^{55,56}$.

However, the MPE for laser pulses is lower than the MPE for continuous laser power, so that lower power levels should be used for pulsed lasers.

For very long viewing (exposure times in the order of several hours) laser safety standards prescribe a power density of $0.01 \mathrm{~W} / \mathrm{m}^{2}$. Figure 1 shows the maximum permissible exposure as a function of exposure time.

In practice some devices achieve this requirement through automatic power adjustment capabilities that insure eye safety at any distance ${ }^{51,57}$. Laser dazzlers with integrated laser range finders have the ability to automatically shut down the beam when it points at a target that lies within the NOHD, and can adjust the laser beam intensity to the target's distance and to the atmospheric conditions between the laser and the target ${ }^{53,58}$

The smart dazzler concept of Upton et al. ${ }^{57}$ and Donne et al. ${ }^{51}$ automatically produces the maximum intensity that is "eye-safe", based on calculations using the intensity based on analysis of an infrared laser probe beam. This analysis provides information on atmospheric attenuation, transmission through dark glasses on the target and light collection of the target. The latter depends on iris aperture, use of binoculars, etc.

During day time the eye has it maximum sensitivity at $555 \mathrm{~nm}$ (photopic vision), while during night time the maximum sensitivity occurs at $510 \mathrm{~nm}$ (scotopic vision). Therefore in the ideal case different wavelengths should be used for day and night operations. The MPE of $25 \mathrm{~W} / \mathrm{m}^{2}$ is in photometric (visual) units: $25 \times 683=1.7 \times 104 \mathrm{~lx}$ in day time and $25 \times 1700=4.3 \times 104 \mathrm{~lx}$ in night time. Figure 2 shows the glare luminance as a result of these two laser powers.

To illustrate the impact of the glare luminance, we can compare it to a computer display that has a luminance between 50 and $300 \mathrm{~cd} / \mathrm{m}^{2}$. This means that a computer display cannot be read within 15 degrees of the laser spot both day and night. Since a perfect diffuse reflector emits $1 / \pi$ units of luminance $\left(\mathrm{cd} / \mathrm{m}^{2}=1 \mathrm{ux} / \mathrm{sr}\right)$ per unit illuminance (lx), we can investigate illumination conditions and see what this means in respect to the glare luminance. Some typical illumination conditions are the following:

- sunlight on an average day ranges from 32,000 to 100,000 lux,

- TV studios are lit at about 1000 lux,

- a bright office has about 400 lux of illumination,

- at sunset and sunrise, ambient outdoor light is also about 400 lux (if the sky is clear),

- moonlight represents about 1 lux,

- starlight measures a mere 0.00005 lux.

It is clear that moonlight with an illumination of $1 \mathrm{~lx}$ is completely overwhelmed by the glare of the laser. For daylight operation, we have a luminance of around $10,000 \mathrm{~cd} / \mathrm{m}^{2}$ with a perfect diffuse reflector that corresponds to less than 5 degrees from the dazzling center. However, more realistic reflector characteristics will lead to higher glare angles. In any case glare or dazzle is much more difficult to achieve in broad daylight. The above is summarized in Figure 2. Dazzling is effective for the angles at which the glare luminance of the laser exceeds the background luminance. The angle which is required to dazzle a scene effectively is scenario dependent ${ }^{59}$. 


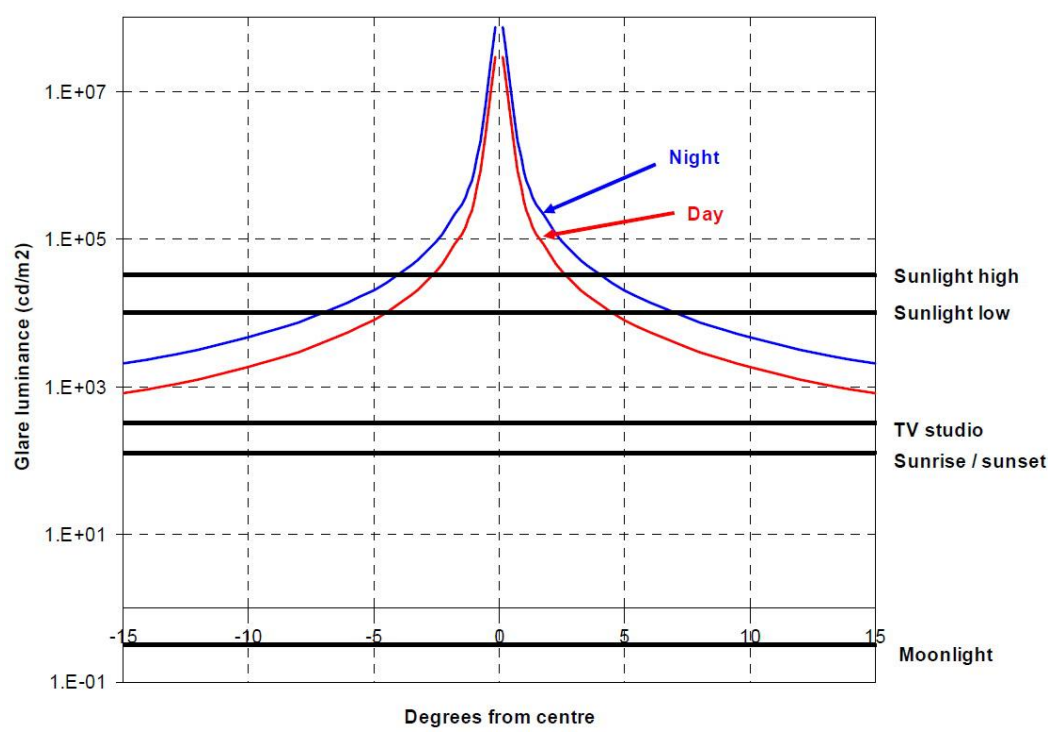

Figure 2. The glare luminance of a laser with maximum permissible power at optimum wavelength during the day and night compared to the luminance of the sun or artificial light sources (assuming the sources are perfect diffuse reflectors).

The illumination during the day by the sun has a maximum illumination level of $10^{5}$ lux. This number can be compared to the maximum laser power of $25 \mathrm{~W} / \mathrm{m}^{2}$, which was obtained from the MPE given by international laser safety standards. An illumination level of $10^{5}$ lux corresponds to $10^{5} / 683=146 \mathrm{~W} / \mathrm{m}^{2}$. This means that the sun produces an illumination level that is a factor 6 above the maximum laser power. It may appear strange that the maximum laser illumination should be less than that of the sun. The reason for this difference is that the sun is a bright disc while a laser is focused to a small point and is therefore more dangerous to the eye.

In conclusion, it can be said that at night-time jamming of human vision can be achieved with dazzle (i.e. glare) or flash blindness well within the safety margins of eye damage. During day-time operation the perspective is not so clear, since considerable laser power at the maximum permissible power has to be applied to generate a sizeable laser glare spot. Since a person can force his eyes to ignore the blink reflex, damage may occur at the power level involved here. If one limits the laser power in the visual bands to eye-safe levels, it will be difficult to achieve a significant dazzling effect at day time. This is due to the fact that eye-safe laser irradiation levels are below the irradiance levels of the sun and result in a low dazzling-to-signal ratio.

\subsection{Requirements for effective flare countermeasures}

Since the sixties flare countermeasures based on chemical combustion (flares) have been used on flying platforms to counter IR (heat seeking) guided missiles ${ }^{60}$. Compositions like MTV (Magnesium/Teflon/Viton) were developed to produce as much IR output as the output of a jet engine. Development is still ongoing for flares mimicking the spectral and flight behavior of aircraft. In this section we will discuss to what extent flares can serve as effective optical countermeasures against CLOS operators. The great advantage of flares over laser systems is that they do not require any pointing and that pyrotechnical devices are already in use on a wide range of platforms, such as flares on aircraft but also smoke grenades on armored vehicles. This makes flares attractive as countermeasures against CLOS operators.

The omnidirectional output of a flare has the advantage of not having to point the energy but at the same time the greatest challenge will be to achieve the required energy output in the direction of the human operator. In addition, a flare can only contain a given amount of chemical energy depending on its geometry and composition. This energy can either be released in a single flash or (for strobing) can be distributed over a series of flashes (and therefore over a longer time period). Moreover, not all the energy contained in the flare will be converted to the desired spectral output. Losses can be expected from heat generation, partial excitation of the actual species that emits this wavelength, and emission in different wavelengths. Depending on the composition and color generating species this loss factor may vary significantly. The energy content of the flare may also vary. The latter can of course be calculated thermodynamically. 
Data about the loss factor are currently not available, especially for the visible wavelength range. These uncertainties prevent a definite answer to the question whether the required output can be reached with a countermeasure based on chemical combustion. Therefore, a very rough estimate will be made based on an ideal case.

Assume that a flash flare device can be made where the energy of a typical MTV flare will be released within a $100 \mathrm{~ms}$ time window. At typical MTV flare produces $2 \mathrm{~kW} / \mathrm{sr}$ over $3 \mathrm{sec}$ in the 3-5 $\mu \mathrm{m}$ band. Assuming that the flare can be approximated by a $2000 \mathrm{~K}$ black-body, a flare producing $2 \mathrm{~kW} / \mathrm{sr}$ in the $3-5 \mu \mathrm{m}$ band, will produce $90 \mathrm{~W} / \mathrm{sr}$ in the visible band between 0.4 to $0.7 \mu \mathrm{m}$. Compressing this output over a $3 \mathrm{sec}$ window into a $0.1 \mathrm{sec}$ window will yield $2700 \mathrm{~W} / \mathrm{sr}$ in the visible band. Of course larger outputs could be expected by burning more material in a flare of multiple flares.

As explained in Section 5.1 at day time an irradiance level at the eye close to the MPE is needed to achieve a countermeasure effect and we will use this value for guidance here. Taking the $25 \mathrm{~W} / \mathrm{m}^{2}$ for a $100 \mathrm{~ms}$ pulse as a reference, we can calculate that a $2700 \mathrm{~W} / \mathrm{sr}$ flare will generate this irradiance at a range of roughly $10 \mathrm{~m}$, ignoring atmospheric transmission losses. Bear in mind that this is the most demanding situation and that under other light conditions flash flares still can have significant effects. For application as a robust countermeasure against CLOS weapons the output of the flares should be significantly increased to achieve useable ranges.

The next challenge is to make a flare that has it's spectral output mostly in the visible range from $530-560 \mathrm{~nm}$ with a maximum at $545 \mathrm{~nm}$. Color generation species need to be generated with a spectral output in this range preferable around the optimum $545 \mathrm{~nm}$. If atomic emission is considered than the element lithium, with a strong emission at $548.5 \mathrm{~nm}$, will be a good candidate. As known from field fireworks, another well-known emitter in this region is $\mathrm{BaCl}$. However, both these emitters have their drawbacks. It will be extremely hard to keep the Li species stable in a reducing flame. Most likely, $\mathrm{LiO}$ is formed which has emission lines in a different range of the spectrum. To generate $\mathrm{BaCl}$ as emitter a chlorine donor needs to be added to the composition, which in most cases will act as a flame retardant, thus causing a decrease in total spectral energy output.

Strobing compositions have been around for years in the fireworks industry. They are available in multiple colors, including green. A wide range of strobing frequencies can be produced, including frequencies in the range from $5-10 \mathrm{~Hz}$ that cause maximal visual discomfort. In addition, flash duration and optical output can be adjusted. Hence, flares appear promising candidates for effective optical countermeasures. However, since airflow affects the burning behavior of flares it may be difficult to exactly control their strobe frequencies in practice ${ }^{61}$.

\subsection{Effects of optical countermeasures on camera systems}

An operator may use a camera instead of direct vision as a counter-countermeasure against flare and laser irradiance. It is therefore of interest to investigate whether this would enable the operator to keep performing his task. To explore this we now apply the same optical countermeasures as described above on a camera operating in the visual band.

For high intensity laser illumination the camera will show a similar effect as the glare effect in the human eye. For cameras the extent of this effect can be described in terms of the diffraction process ${ }^{62}$. Through diffraction the laser illuminates an area around the central laser spot in the image and the ratio between additional laser illumination and background is fixed. Reducing camera sensitivity to avoid the saturation by the laser will not increase the contrast because the background intensity will be reduced at the same rate. Figures 3a-c show camera images for a typical CCD camera $(752 \times 82$ pixels, $f=25 \mathrm{~mm}, \mathrm{f} / 1.4)$ and irradiance levels corresponding roughly to the irradiance levels indicated in Table 2 corresponding to startle, glare and afterimages respectively.

For the flash frequency effects the recovery time of the camera will become of interest. The recovery time of the camera depends on the intensity of the illumination. Figure 4 shows the recovery time as a function of frame number after switching off a laser with a given irradiance for the same CCD camera with a $25 \mathrm{~Hz}$ frame rate. The results are summarized in Table 3. 


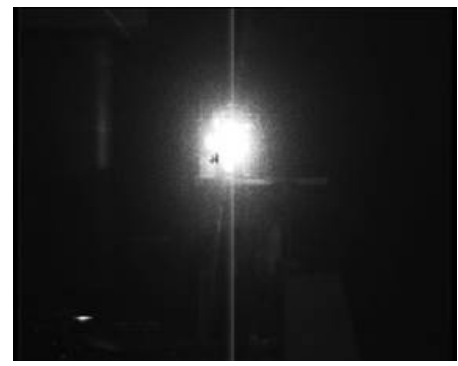

$5.6 \times 10^{4} \mathrm{pW} / \mathrm{cm}^{2}$

$\left(\right.$ Startle $\left.5 \times 10^{-8} \mathrm{~W} / \mathrm{cm}^{2}\right)$

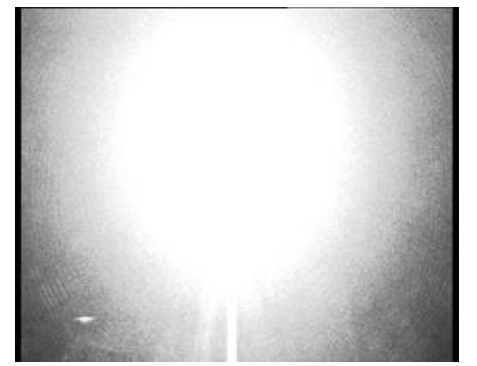

$2.1 \times 10^{6} \mathrm{pW} / \mathrm{cm}^{2}$

(Glare $5 \times 10^{-6} \mathrm{~W} / \mathrm{cm}^{2}$ )

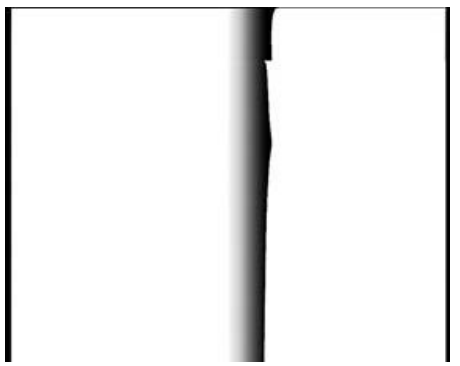

$1.1 \times 10^{8} \mathrm{pW} / \mathrm{cm}^{2} \mathrm{~cm}$

(Afterimages $10^{-4} \mathrm{~W} / \mathrm{cm}^{2}$ )

Figure 3. CCD camera images at irradiance levels corresponding roughly to the irradiance levels that cause respectively startle, glare and afterimages in the human visual system.

Table 3. CCD camera recovery time (expressed as the number of frames) after switching off a laser with a given irradiance.

\begin{tabular}{|lll|}
\hline \multicolumn{2}{|l|}{ Laser irradiance } & Recovery time \\
\hline $10^{5} \mathrm{pW} / \mathrm{cm}^{2}$ & (laser spot diameter 200 pixels) & Within one frame \\
$10^{6} \mathrm{pW} / \mathrm{cm}^{2}$ & (laser spot diameter 500 pixels & Within one frame \\
$10^{7} \mathrm{pW} / \mathrm{cm}^{2}$ & (all pixels saturated) & Within 15 frames \\
$10^{8} \mathrm{pW} / \mathrm{cm}^{2}$ & (camera all black) & Within 20 frames \\
\hline
\end{tabular}
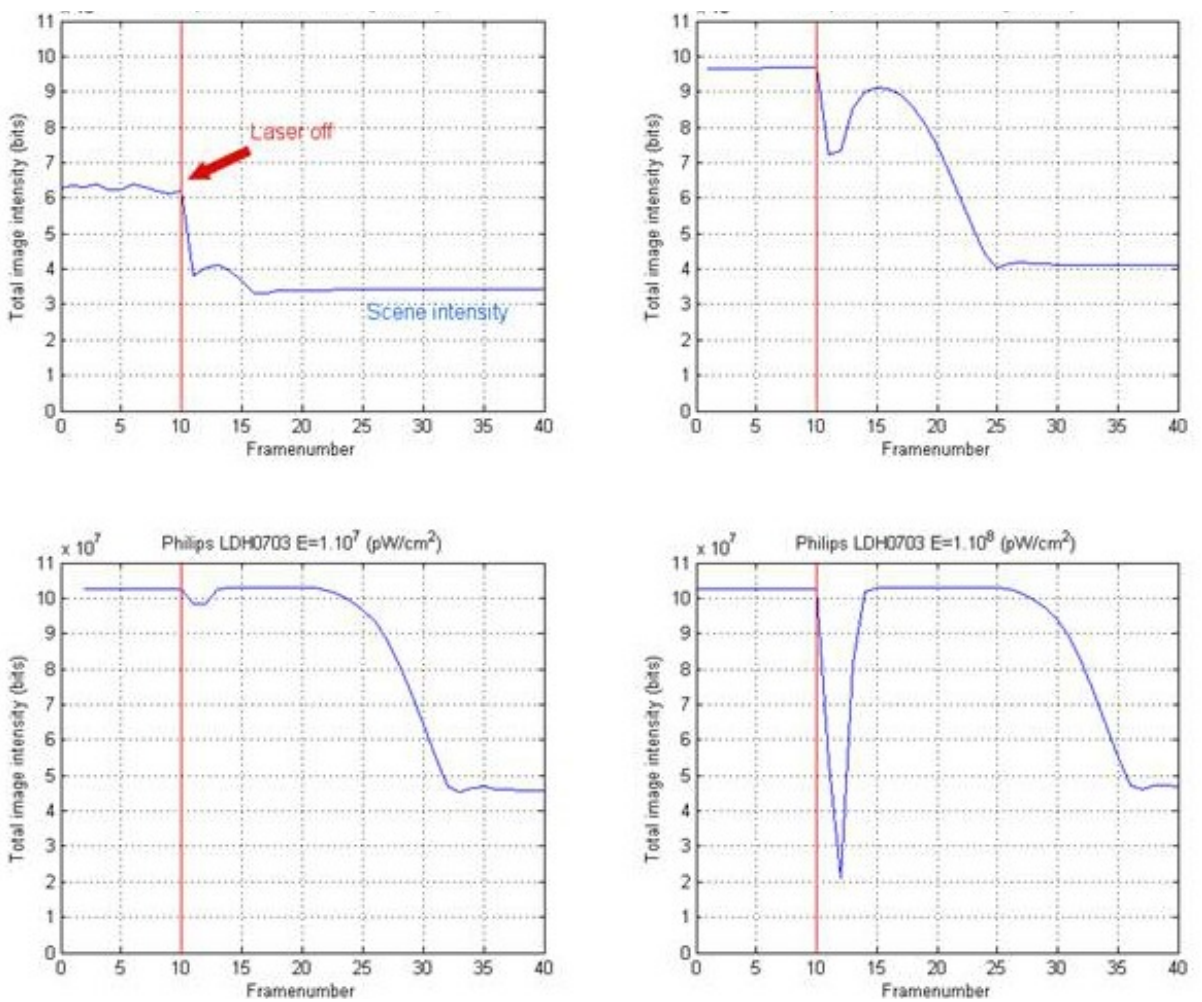

Figure 4. Recovery time (expressed as the number of frames) of a CCD camera with a $25 \mathrm{~Hz}$ frame rate as a after switching off a laser with a given irradiance. 
For the optimum flicker frequencies in the range between $5-15 \mathrm{~Hz}$ and a $25 \mathrm{~Hz}$ camera frame rate not all the images will be illuminated and a beating effect will occur. Also the synchronization of the laser pulse and the camera stare time need to be in phase to achieve optimum disturbance. Longer laser pulses will have a higher probability to "hit" the period when the camera captures the image. Figure 5 illustrates the number of illuminated frames per second as a function of laser frequency for a laser duty cycle of $25 \%$. The different curves are for different integration or stare times of the camera. As expected the hit rate for short stare times is much smaller than for long stare times. Note that for example a 5 $\mathrm{Hz}$ illumination can still hit more than 8 frames per second because one long pulse can hit more than one frame. The spikes in the curves are caused by phase difference effects of the CCD frame rate and the illuminating laser frequency.

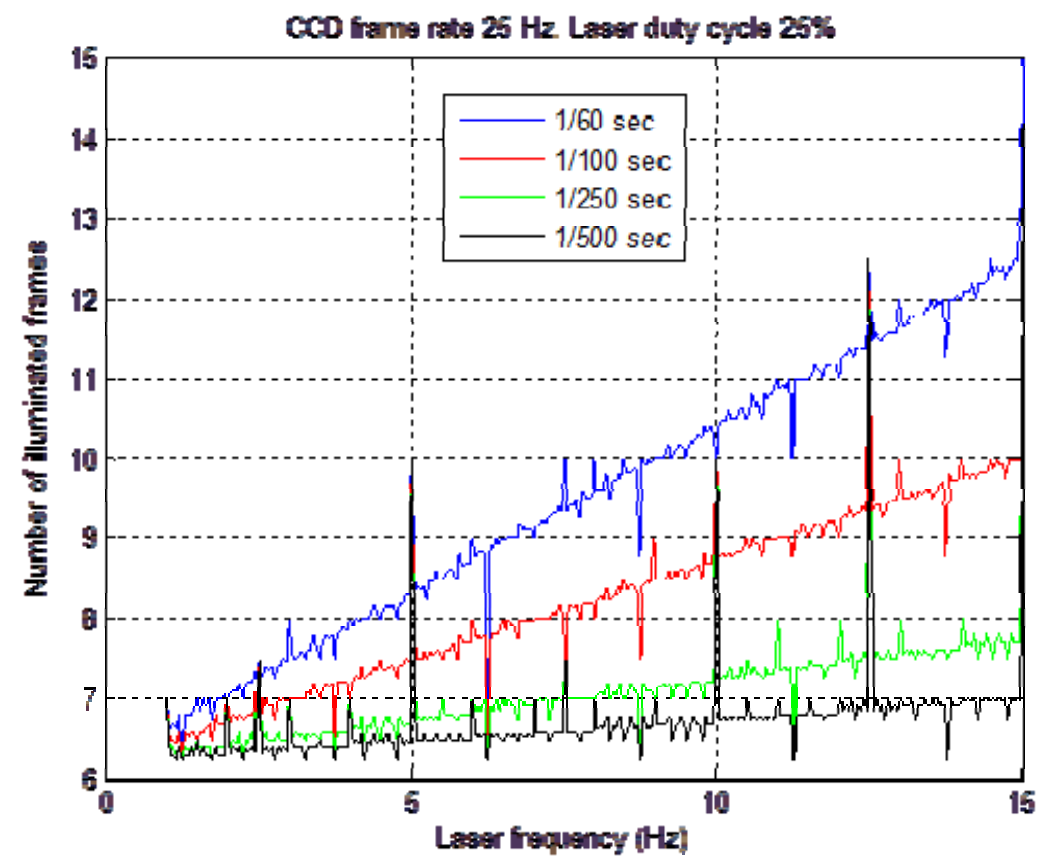

Figure 5. Number of illuminated frames per second as a function of laser frequency for a laser duty cycle of $25 \%$.

\section{CONCLUSIONS}

In summary, we have presented an overview of the literature on the visual effects of bright lights on the human eye. From this overview we identified three types of potential robust countermeasures against optically guided weapon system operators:

1. a high intensity flash - causing temporary flash blindness,

2. a bright light source - causing contrast reduction,

3. a flickering pattern of light - causing contrast reduction.

We then discussed the actual requirements for effective and robust deployment of each of these approaches. We demonstrated that lasers can achieve these requirements relatively easy, but pointing them at a CLOS operator is complicated. Flares are more attractive to use as optical countermeasures, but they need further development to meet the requirements. Finally we also discussed to what extent mitigation measures by the weapon operator might reduce the effectiveness of the optical countermeasures. 


\section{REFERENCES}

[1] Laser Emitters and Flight Safety Study Group, "Laser bioeffects and their hazards to flight operations," In: C. CurdtChristiansen (Ed.), Manual on Laser Emitters and Flight Safety, pp. 3.1-3.16, International Civil Aviation Organization, Montreal, CA, 2001.

[2] Svec, J. and Beer, D., "Directed energy in the military environment," Leading Edge, 7(4) ,pp. 74-77, 2012.

[3] CIE. "CIE Equations for disability glare", CIE 146 TC 1-50, International Commission on Illumination CIE, Vienna, Austria, 2002.

[4] Stiles, W.S., "The effect of glare on the brightness threshold," Proceedings of the Royal Society of London B, 104 ,pp. 322-351, 1929.

[5] Holladay, L.L., "The fundamentals of glare and visibility," Journal of the Optical Society of America, 12 ,pp. 271$319,1926$.

[6] Vos, J.J., Walraven, J. and van Meeteren, A., "Light profiles of the foveal image of a point source," Vision Research, 16(2), pp. 215-219, 1976.

[7] Chisum, G.T., "Flashblindness recovery following exposure to constant energy adaptive flashes," Aerospace Medicine, 44(4) ,pp. 407-413, 1971.

[8] Blackwell, O.M. and Blackwell, H.R., "Individual responses to lighting parameters for a population of 235 observers of varying ages," Journal of the Illuminating Research Society, 2 ,pp. 205-232, 1980.

[9] Ward, B., Bowie, W.H. and Cushman, W.H., "Flashblindness recovery with and without protection in simulated flight conditions," Aerospace Medicine, 42(2) ,pp. 149-152, 1971.

[10] Wang, L., Söderberg, P.G. and Tengroth, B., "Measurement of visual readaptation time after flash exposure using optokinetic nystagmus," Acta Ophthalmologica, 71(6) ,pp. 810-818, 1993.

[11] Wütrich, S., Schmid, M., Lüthy, W. and Weber, H.P., "Recovery time of the human eye after exposure to laser light," In: S.K. Park \& R.D. Juday (Ed.), Visual Information Processing VI,SPIE-3074, pp. 2-12, The International Society for Optical Engineering, Bellingham, WA, 1997.

[12] Smith, P.A., "The transient effects of visible laser light on visual function," In: Laser induced acute visual and cognitive incapacitation of aircrew, protection management, and cockpit integration,AGARD-AR-354, pp. 19-24, North Atlantic Treaty Organization, Neuilly-sur-Seine, France, 1996.

[13] Davson, H.Ed., "The Eye. Vol. 2A: Visual function in man (2nd ed.)", Academic Press, New York, London, San Francisco, 1976.

[14] Megaw, E., "The visual environment," In: Handbook of Human Performance,Vol. 1, pp. 261-296, Academic Press, 1992.

[15] Sliney, D.H. and Wolbarsht, M., "Safety with lasers and other optical sources: A comprehensive handbook", Plenum Press, New York, USA, 1980.

[16] Belkin, M., "Ophthalmic manifestations of laser-induced eye injuries," In: B.E. Stuck \& M. Belkin (Ed.), Laserinflicted eye injuries: Epidemiology, prevention, and treatment,SPIE 2674, pp. 2-6, The International Society for Optical Engineering, Bellingham, MA, USA, 1996.

[17] Hudson, S.J., "Eye injuries from laser exposure: a review," Aviation, Space and Environmental Medicine, 69(5) ,pp. 519-524, 1998.

[18] United Nations. "Protocol on Blinding Laser Weapons (Protocol IV to the 1980 United Nations Convention on Certain Conventional Weapons)", United Nations, Vienna, Austria, 1995.

[19] Saito, M. \& Narisada, K. "The effect of flickering light on visual comfort (in Japanese)", National Technical Report 14-1, 1968.

[20] Narisada, K. and Schreuder, D., "Light pollution handbook", Springer, Dordrecht, The Netherlands, 2004.

[21] Collins, J.B. and Hopkinson, R.G., "Intermittent light stimulation and flicker sensation," Ergonomics, 1(1) ,pp. 61$76,1957$.

[22] Howett, G.L., Kelly, K.L. \& Pierce, E.T. "Emergency vehicle warning lights: state of the art", NBS Special Publication 480-16, National Bureau of Standards, Washington, DC, 1978.

[23] Grüsser, O.-J. and Creutzfeldt, O.D., "Neurophysiological basis of Brucke-Bartley effect; maxima of impulse frequency of retinal and cortical neurons in flickering light of middle frequency," Pflügers Archiv: European journal of physiology, 263(6) ,pp. 668-681, 1957.

[24] Bartley, S.H. and Nelson, T.H., "Further study of pulse-to-cycle fraction and flicker frequency," Journal of the Optical Society of America, 51(1) ,pp. 41-45, 1961. 
[25] Alferdinck, J.W.A.M., "Traffic safety aspects of high-intensity discharge headlamps: discomfort glare and direction indicator conspicuity," In: A.G. Gale, I.D. Brown, C.M. Haslegrave \& S.P. Taylor (Ed.), Vision in Vehicles V, pp. 337-344, Elsevier, Amsterdam, 1996.

[26] Sivak, M., Simmons, C.K. and Flannagan, M., "Effect of headlamp area on discomfort glare," Lighting Research and Technology, 22(1) ,pp. 49-52, 1990.

[27] Alferdinck, J.W.A.M., Kriekaard, J.J. \& Toet, A. "Assessment of chromatic flicker effects on human task performance", TNO-DV 2010 A104, TNO Defense, Security and Safety, Soesterberg, The Netherlands, 2010.

[28] Stamper, D.A., Lund, D.J., Molchany, J.W. and Stuck, B.E., "Laser flash effects on tracking performance and the aversion response," In: B.E. Stuck \& M. Belkin (Ed.), Laser and Noncoherent Light Ocular Effects: Epidemiology, Prevention, and Treatment,SPIE 4246, pp. 71-77, The International Society for Optical Engineering, Bellingham, MA, USA, 2001.

[29] Rubtsov,V. (2007). L.T.C.Optech Ventures. Incapacitating Flashing Light Apparatus and Method. US Patent \# $7,180,426$.

[30] Harding, G.F.A. and Jeavons, P.M., "Photosensitive epilepsy", MacKeith Press, London, UK, 1994.

[31] Zifkin, B.G. and Inoue, Y., "Visual reflex seizures induced by complex stimuli," Epilepsia, 45(S1) ,pp. 27-29, 2004.

[32] De Bittencourt, P.R.M., "Photosensitivity: The magnitude of the problem," Epilepsia, 45(S1) ,pp. 30-34, 2004.

[33] Fisher, R.S., Harding, G., Erba, G., Barkley, G.L. and Wilkins, A., "Photic- and pattern-induced seizures: a review for the Epilepsy Foundation of America Working Group," Epilepsia, 46(9) ,pp. 1426-1441, 2005.

[34] Bhattacharya, J., Watanabe, K. and Shimojo, S., "Nonlinear dynamics of evoked neuromagnetic responses signifies potential defensive mechanisms against photosensitivity," International Journal of Bifurcation and Chaos, 14(8) ,pp. 2701-2720, 2004.

[35] Watanabe, K., Imada, T., Niheui, K. and Shimojo, S., "Neuromagnetic responses to chromatic flicker: implications for photosensitivity," Neuroreport, 13(16) ,pp. 2161-2165, 2002.

[36] Parra, J., Lopes da Silva, F.H., Stroink, H. and Kalitzin, S., "Is colour modulation an independent factor in human visual photosensitivity?," Brain, 130(6) ,pp. 1679-1689, 2007.

[37] Shirakawa, S., Funatsuka, M., Osawa, M., Fujita, M. and Oguni, H., "A study of the effect of color photostimulation from a cathode-ray tube (CRT) display on photosensitive patients: the effect of alternating redcyan flicker stimulation," Epilepsia, 42(7) ,pp. 922-929, 2001.

[38] Yamasaki, T., Goto, Y., Kinukawa, N. and Tobimatsu, S., "Neural basis of photo/chromatic sensitivity in adolescence," Epilepsia, 49(9) ,pp. 1611-1618, 2008.

[39] Klistorner, A., Crewther, D.P. and Crewther, S.G., "Temporal analysis of the chromatic flash VEP--separate colour and luminance contrast components," Vision Research, 38(24) ,pp. 3979-4000, 1998.

[40] Yamasaki, T., Goto, Y. and Tobimatsu, S., "Can we estimate the activating effects of visual channels in primary visual cortex by flicker VEPs?," International Congress Series, 1278(March) ,pp. 73-76, 2005.

[41] McAleer,M.K., Zollars,B.G., Durrett,M.G., Jamison,K.D., Mayo,M.W., \& Mitchell,D.R. (2008). I.Nanohmics. Non-lethal projectile for disorienting adversaries. United States Patent Application. US 2008/0216699 A1,

[42] Tocci,N.C., Cramer,EJ., Tocci,M.D., \& German,J.D. (2001). Science \& Engineering Associates Inc. Enhanced non-lethal visual security device. US Patent 6190022. No. 409328 filed on 09/30/1999,

[43] Hambling, D., "Non-lethal beams target the eyes," New Scientist, 198(2655) ,pp. 38-40, 2008.

[44] Stamper, D.A., Lund, D.J., Molchany, J.W. and Stuck, B.E., "Human pupil and eyelid response to intense laser light: implications for protection," Perceptual and Motor Skills, 95 ,pp. 775-782, 2002.

[45] Andreassi, J.L., "Psychophysiology. Human behavior \& physiological response (4th ed.)", Lawrence Erlbaum Associates, Mahwah, NJ, USA, 2000.

[46] Svensson, S., Björkert, S., Kariis, H. and Lopes, C., "Countering laser pointer threats to road safety," In: C. Lewis \& G.P. Owen (Ed.), Optics and Photonics for Counterterrorism and Crime Fighting II,SPIE-6402, pp. 640207640207, The International Society for Optical Engineering, Bellingham, WA, 2006.

[47] Thomas, S.R., "Aircrew laser eye protection: visual consequences and mission performance," In: Proceedings of the 31st Annual Symposium of the SAFE Association, pp. 298-313, SAFE Association, Yoncalla, Oregon, 1994.

[48] SAE. "Safety considerations for high-intensity lights (HIL) directed into the navigable airspace", ARP5560, SAE International, Warrendale, PA, 2008.

[49] American National Standards Institute (2005). American national standard for the safe use of lasers outdoors (Standard Z136.6). New York, USA: The Laser Institute of America.

[50] Arecchi, A.V., Messadi, T. and Koshel, R.J., "Field Guide to Illumination", SPIE Press, Bellingham, WA, 2007. 
[51] Donne, G.A., Hauck, J.P., Ludman, J., Moldow, R., Servatius, R. and Yagrich, K., "Multi-wavelength optical dazzler for personnel and sensor incapacitation," In: G.T. Shweary, J.G. Blitch \& C. Land (Ed.), Enabling Technologies and Design of Nonlethal Weapons,SPIE-6219, pp. 9-18, The International Society for Optical Engineering, Bellingham, WA, 2006.

[52] Beer, J.M. and Freeman, D.A., "Lasers' spectral and temporal profile can affect visual glare disability," Aviation, Space, and Environmental Medicine, 83(12),pp. 1135-1144, 2012.

[53] Hecht, J., "Photonic frontiers: Nonlethal lasers. Nonlethal lasers deter attacks and warn away noncombatants," Laser Focus World, 04/01/2013(available from www.laserfocusworld.com), 2013.

[54] Griffioen-Young, H.J., Alferdinck, J.W.A.M., Houtgast, T. \& van Bree, J.L.M.J. "The human response to sound, light, and rubber pellets: Theory behind non-lethal hand grenades", Report TM-99-A031, TNO Human Factors Research Institute, Soesterberg, The Netherlands, 1999.

[55] American National Standards Institute (2005). American national standard for the safe use of lasers (Standard Z136.6). New York, USA: The Laser Institute of America.

[56] IEC (2001). Safety of laser products, Part 1: Equipment classification, requirements and user's guide (IEC 608251:2001). Geneva, Switzerland: International Electronic Commission.

[57] Upton, T.D., Ludman, J.E. and Watt, D.W., "Smart white-light dazzler," In: E.M. Carapezza (Ed.), Sensors, and Command, Control, Communications, and Intelligence (C3I) Technologies for Homeland Security and Homeland Defense III,SPIE-5403, pp. 493-501, The International Society for Optical Engineering, Bellingham, WA., USA, 2004.

[58] Beer, J., "Laser light stimuli for maritime warning and repulsion: Evolving concepts and capabilities," In: Procedings of the 2010 International Waterside Security Conference (WSS), pp. 1-3, IEEE, Piscataway, NJ, USA, 2010.

[59] van den Heuvel, J.C., Toet, A. \& Rieter-Barrell, Y. "Effects of optical devices on humans", TNO-DV 2009 A435, TNO Defense, Security and Safety, The Hague, The Netherlands, 2009.

[60] Douda, B.E. "Genesis of infrared decoy flares. The early years from 1950 into the 1970s", Report nr. NSWCCR/RDTR-08/63, Naval Surface Warfare Center, Crane Division, Crane, Indiana USA, 2009.

[61] Corbel, J.M.L. (2013). New insights into strobe reactions: An intriguing oscillatory combustion phenomenon. Utrecht, The Netherlands: Utrecht University.

[62] Schleijpen, R.M.A., Dimmeler, A., Eberle, B., van den Heuvel, J.C., Mieremet, A.L., Bekman, H. and Mellier, B., "Laser dazzling of focal plane array cameras," In: D.H. Titterton \& M.A. Richardson (Ed.), Technologies for Optical Countermeasures IV,SPIE-6738, pp. 0O-1-10, The International Society for Optical Engineering, Bellingham, WA, 2007. 